\title{
Estimation and Prediction for Pareto Distribution under Type-II Progressive Hybrid Censoring Scheme
}

\author{
M. M. Mohie El-Din \\ Department of Mathematics, \\ Faculty of Science, \\ Al-Azhar University, Egypt \\ Marwa M. Mohie El-Din \\ Russian University, \\ Cairo, Egypt
}

\author{
A. Sadek \\ Department of Mathematics, \\ Faculty of Science, \\ Al-Azhar University, Egypt \\ M. Nagy \\ Department of Mathematics, \\ Faculty of Science, \\ Fayoum University, Egypt
}

\begin{abstract}
In this paper, the maximum likelihood and Bayesian estimation are developed based on Type-II progressive hybrid censoring scheme from the Pareto distribution. One and twosample Bayesian prediction is also discussed using Type-II progressive hybrid censoring scheme. Finally, numerical example is presented for illustrating all the inferential procedures developed here.
\end{abstract}

\section{Keywords}

Bayesian estimation, Bayesian prediction, Pareto distribution, Maximum likelihood estimation, Type-II progressive hybrid censoring sample

\section{INTRODUCTION}

In reliability analysis, experiments are often terminated before all units on test fail based on cost and time considerations. In such cases, failure information is available only on part of the sample, and only partial information on all units that had not failed. Such data are called censored data. There are several forms of censored data. Two commonly used right censoring schemes are the Type-I and Type-II censoring. Consider $n$ identical units on a life-testing experiment, in the Type-I censoring scheme, the experiment is terminated when a prefixed censoring time $T$ is reached. In the Type-II censoring scheme, the experiment gets terminated when a pre-specified number $m \leq n$ of failures is observed, a natural extension of Type-II censoring scheme will be a censoring scheme in which $n-m$ units are withdrawn from the life-test at different time points (rather than all at the final time point $\left.X_{m: n}\right)$. Such a versatile censoring scheme is referred to as progressive Type-II right censoring. Under this scheme, $n$ units are placed on a life-testing experiment and only $\mathrm{m}$ complete failures are to be observed. When the first failure is observed, $R_{1}$ of the $n-1$ surviving units are randomly selected and removed. At the second observed failure, $R_{2}$ of the $n-R_{1}-2$ surviving units are randomly selected and removed. The experiment finally terminates at the time of the $m^{\text {th }}$ failure when all remaining $R_{m}=n-R_{1}-\cdots-R_{m-1}-$ $m$ surviving units are removed. The censoring numbers $R_{i}$ are fixed prior to the experiment. The $m$ ordered failure times thus observed will denote by $X_{1: m: n}, \ldots, X_{m: m: n}$ it is clear that $n=m+\sum_{k=1}^{m} R_{k}$. The resulting $\mathrm{m}$ ordered values which are obtained from this type of censoring are referred to as Type-II progressive right censored order statistics. Several authors have studied progressive Type-II censoring and properties of order statistics arising from such a progressive censored life test. Some key references are [1], [2] and [3].
Childs et al. in [4], proposed another hybrid censoring scheme, the Type-II progressive hybrid censoring scheme (Type-II PHCS), that would terminate the experiment at the random time $\max \left(X_{m: m: n}, T\right)$ where $T \in(0, \infty)$ and $1 \leq m \leq$ $n$ with progressive censoring scheme $R=\left(R_{1}, \ldots, R_{m}\right)$ are fixed in advance. This new progressive hybrid censoring scheme, guarantees that at least $\mathrm{m}$ failure times will be observed. For the case $X_{m: m: n}<T$ will not only follow the pre-specified scheme to remove the units after each failure, but continue to observe failures (without any further withdrawals) up to time $T$. Let $D$ denote the number of failures that occur before time $T$. Then, the progressive censoring scheme in this case will become $R^{*}=$ $\left(R_{1}, \ldots, R_{m}, R_{m+1}, \ldots, R_{D}, R_{\tau}^{*}\right)$, where $R_{i}=0$ for $i=m, m+$ $1, \ldots, D$. Clearly, the termination time under this scheme is unknown to the experimenter which is a disadvantage, but there is an advantage that more than $\mathrm{m}$ failures may be observed which will greatly increase the efficiency of the statistical analysis (see Fig. 1).

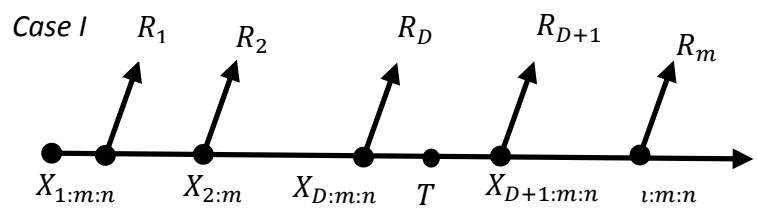

Case

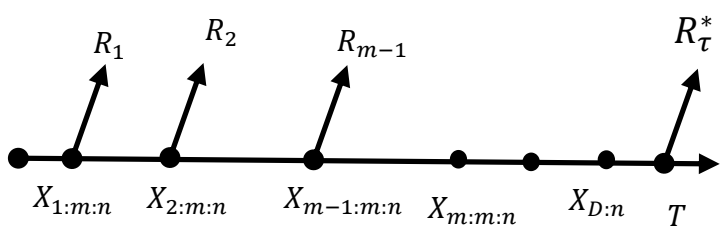

Fig. 1. Schematic representation of adaptive progressive Type-II censoring scheme

In this paper, the underlying distribution is assumed to be the Pareto distribution which introduced by Pareto in [5], as a model for the distribution of income, with the probability density function $(P D F)$ and cumulative distribution function $(C D F)$ as

$$
f(x \mid \alpha, \beta)=\frac{\alpha}{x} \exp \left[-\alpha \log \left(\frac{x}{\beta}\right)\right], x \geq \beta,
$$

and 


$$
F(x \mid \alpha, \beta)=\left(\frac{\beta}{x}\right)^{\alpha}
$$

where $\alpha, \beta>0$.

In recent years, its models in several different forms have been studied by many authors including [6], [7] and [8] among others.

The rest of this paper is organized as follows. In Section 2, the description of the model of the Type-II PHCS is presented. The maximum likelihood ML estimator and the Bayesian estimators under the squared error loss function for the unknown parameters are derived in Section 3. In Section 4, we derive the Bayesian prediction for the failure times of all units that are removed in all stages of censoring. Bayesian prediction for progressive order statistics from an unobserved future sample from the same distribution is derived in Section 5. Finally, in Section 6, for illustrating all the inferential methods developed here, numerical example is presented.

\section{THE MODEL DESCRIPTION}

Under Type-II PHCS described above, we have one of the following two cases of observations:

1. Suppose that the $m^{\text {th }}$ failure occurs after $T$ (i.e. $T<X_{m: m: n}$ ), then the experiment terminates at $X_{m: m: n}$ and we will observe $\left\{X_{1: m: n}<\cdots<\right.$ $\left.X_{m: m: n}\right\}$.

2. Suppose that the $m^{\text {th }}$ failure occurs before T (i.e. $\left.X_{m: m: n} \leq T\right)$, then the experiment terminates at $T$ and we will observe $\left\{X_{1: m: n}<\cdots<\right.$ $\left.X_{m: m: n}<X_{m+1: n}<\cdots<X_{D: n}\right\}$.

Thus, the joint density function based on the above cases can be written as:

$$
\begin{aligned}
& f_{\underline{\boldsymbol{X}}}(\underline{\boldsymbol{X}}) \\
& =\left[\prod_{i=1}^{D^{*}} \sum_{j=1}^{m} R_{j}^{*}\right]\left[\prod_{i=1}^{D^{*}} f\left(x_{i: m: n}\right)\left[\bar{F}\left(x_{i: m: n}\right)\right]^{R_{i}^{*}}\right][\bar{F}(T)]^{R_{\tau}^{*}}
\end{aligned}
$$

where

$$
\begin{gathered}
D^{*}=\left\{\begin{array}{cc}
m & T<X_{m: m: n}, \\
D & X_{m: m: n} \leq T,
\end{array}\right. \\
R^{*}=\left\{\begin{array}{l}
\left(R_{1}, \ldots, R_{\tau}^{*}, \ldots, R_{m}\right) \quad \text { if } T<X_{m: m: n}, \\
\left(R_{1}, \ldots, R_{m-1}, 0, \ldots, 0, R_{\tau}^{*}\right) \text { if } X_{m: m: n} \leq T,
\end{array}\right.
\end{gathered}
$$

with $R_{\tau}^{*}$ is the number of surviving units that are removed at $T$, given by

$$
R_{\tau}^{*}= \begin{cases}0 & \text { if } T<X_{m: m: n}, \\ n-D-\sum_{j=1}^{m-1} R_{j} & \text { if } X_{m: m: n} \leq T,\end{cases}
$$

with

$$
\underline{\boldsymbol{x}}=\left\{\begin{array}{lr}
\left(x_{1: m: n}, \ldots x_{m: m: n}\right) & \text { if } T<X_{m: m: n}, \\
\left(x_{1: m: n}, \ldots x_{m: m: n}, x_{m+1: n} \ldots, x_{D: n}\right) & \text { if } X_{m: m: n} \leq T,
\end{array}\right.
$$

Upon using (1.1) and (1.2) in (2.1), the likelihood function of $\alpha, \beta$ based on Type-II PHCS can be obtained as

$$
\begin{gathered}
L(\alpha, \beta ; \underline{\boldsymbol{X}})=\left[\prod_{i=1}^{D^{*}} \sum_{j=1}^{m} R_{j}^{*}\right]^{D^{*}}\left(\prod_{i=1}^{D^{*}} \frac{1}{x_{i}}\right) \exp \{-\alpha[W(\underline{\boldsymbol{x}}, \beta) \\
+n \log \beta]\}
\end{gathered}
$$

where

$$
W(\underline{\boldsymbol{x}})=\sum_{i=1}^{D^{*}}\left(R_{i}^{*}+1\right) \log x_{i: n}+R_{\tau}^{*} \log T .
$$

\section{THE ML AND BAYESIAN ESTIMATIONS}

It is clear that the likelihood function is monotone increasing function in $\beta$, so its maximum value $\hat{\beta}_{M L}$ will be attained at the maximum value $x_{1}$ of $\beta$.

From (2.6), The log-likelihood function of $(\alpha, \beta)$ is given by

$$
\log [L(\alpha, \beta \mid x)] \propto D^{*} \log \alpha-\alpha[W(x)-n \log \beta],
$$

to maximize relative to $\alpha$, differentiate (3.1) with respect to $\alpha$ and solve the equation

$$
\frac{\partial \log [L(\alpha, \beta \mid \underline{\boldsymbol{x}})]}{\partial \alpha}=0,
$$

so, the ML estimator of $\alpha$ is obtained as

$$
\hat{\alpha}_{M L}=\frac{D^{*}}{W(\underline{\boldsymbol{x}})+n \log x_{1}} .
$$

For the Bayesian Estimations, under the assumption that both parameters $\alpha$ and $\beta$ are unknown, we may consider the joint prior density function of $\alpha$ and $\beta$ which was suggested by Lwin in [9] and generalized by Arnold and Press in [10]. The generalized Lwin prior or the power-gamma prior is given by

$$
\pi(\alpha, \beta ; \delta) \propto \alpha^{a} \beta^{-1} \exp [-\alpha(\log c-b \log \beta)],
$$

where $\alpha>0,0<\beta<d, a, b, c$ and $d$ are positive constants and $d^{b}<c$.

Upon combining (2.6) and (3.3), given Type-II PHCS , the posterior density function of $\alpha, \beta$ is obtained as

$$
\begin{aligned}
\pi^{*}(\alpha, \beta \mid \underline{\boldsymbol{x}}) & =\frac{L(\alpha, \beta \mid \underline{\boldsymbol{x}}) \pi(\alpha, \beta)}{\int_{0}^{x_{0}} \int_{0}^{\infty} L(\alpha, \beta \mid \underline{\boldsymbol{x}}) \pi(\alpha, \beta) d \alpha d \beta} \\
& =I^{-1} \alpha^{D^{*}+a} \beta^{-1} \exp \{-\alpha[W(\underline{\boldsymbol{x}}) \\
& -(n+b) \log \beta+\log c]\},
\end{aligned}
$$

where

$$
\begin{aligned}
I & =\int_{0}^{x_{0}} \int_{0}^{\infty} \alpha^{D^{*}+a} \beta^{-1} \exp \{-\alpha[W(\underline{\boldsymbol{x}})-(n+b) \log \beta \\
& \left.=\frac{\Gamma\left(D^{*}+a\right)}{n+b}[W(\underline{\operatorname{x}})-(n+b) \log c]\right\}
\end{aligned}
$$

with $x_{0}=\min \left(x_{1: m: n}, d\right)$.

By using (3.4), the Bayesian estimator of $\alpha$ under the squared error loss function is the mean of the posterior density function, given by

$$
\hat{\alpha}_{B}=\int_{0}^{x_{0}} \int_{0}^{\infty} \alpha \pi^{*}(\alpha, \beta \mid \underline{x}) d \alpha d \beta
$$

Hence, the Bayesian estimator of $\alpha$ under the squared error loss function is obtained as

$$
\hat{\alpha}_{B}=\frac{D^{*}+a}{W(\underline{\boldsymbol{x}})-(n+b) \log x_{0}+\log c}
$$

and the Bayesian estimator of $\beta$ under the squared error loss function is obtained as 


$$
\begin{aligned}
\hat{\beta}_{B}= & \int_{0}^{x_{0}} \int_{0}^{\infty} \beta \pi^{*}(\alpha, \beta \mid \underline{\boldsymbol{x}}) d \alpha d \beta, \\
= & I^{-1} x_{0} \int_{0}^{\infty} \frac{\alpha^{D^{*}+a}}{\alpha(n+b)+1} \exp \{-\alpha[W(\underline{\boldsymbol{x}}) \\
& \left.\left.-(n+b) \log x_{0}+\log c\right]\right\} d \alpha \\
= & \frac{I^{-1} x_{0}}{(n+b)}\left[W(\underline{\boldsymbol{x}})-(n+b) \log x_{0}+\log c\right]^{-\left(D^{*}+a\right)} \\
& \times \int_{0}^{\infty} \frac{t^{D^{*}+a} e^{-t}}{t+\left[W(\underline{\boldsymbol{x}})-(n+b) \log x_{0}+\log c\right] /(n+b)} d t \\
& =\left(\frac{x_{0}}{\Gamma\left(D^{*}+a\right)}\right) \Phi\left(D^{*}\right. \\
& \left.+a,\left(\frac{\left[W_{2}(\underline{\boldsymbol{x}})-(n+b) \log x_{0}+\log c\right]}{(n+b)}\right)\right)
\end{aligned}
$$

where

$$
\Phi(x, y)=\int_{0}^{\infty}\left(\frac{t^{x} e^{-t}}{t+y}\right) d t
$$

A partial tabulation of $\psi(x, y)=\left(\frac{y}{\Gamma(x)}\right) \Phi(x-1, y)$ has been provided by Arnold and Press in [10].

\section{ONE-SAMPLE BAYESIAN PREDICTION}

For $\rho=1,2, \ldots, R_{j}^{*}$, let $X_{\rho: R_{j}^{*}}$ denote the $\rho^{\text {th }}$ order statistic out of $R_{j}^{*}$ removed units at stage $j$, and $j=1,2, \ldots, D^{*}$. Then, the conditional density function of $X_{\rho: R_{j}^{*}}$, given the observed progressive Type-II censoring scheme $x_{1: n}, x_{2: n}, \ldots, x_{D^{*}: n}$, is given by Basak et al. in [11] as follows:

$$
\begin{aligned}
f_{\boldsymbol{X}_{\boldsymbol{\rho}: R_{j}^{*}}(x \mid \underline{\boldsymbol{x}})} & =f(x \mid \underline{\boldsymbol{x}}) \\
& =\frac{R_{j}^{*} !}{(\rho-1) !\left(R_{j}^{*}-\rho\right) !} \\
& \times \frac{\left[F(x)-F\left(x_{j}\right)\right]^{\rho-1}[\bar{F}(x)]^{R_{j}^{*}-\rho} f(x)}{\left[\bar{F}\left(x_{j}\right)\right]^{R_{j}^{*}}}
\end{aligned}
$$

where

with $\boldsymbol{x}_{\boldsymbol{\tau}}=\boldsymbol{T}$.

$$
j= \begin{cases}1, \ldots, m & \text { if } T<X_{m: m: n} \\ 1, \ldots, m-1, \tau & \text { if } X_{m: m: n} \leq T,\end{cases}
$$

By using (1.1) and (1.2) in (4.1), the conditional density function of $\boldsymbol{X}$, given progressive Type-II censoring scheme, is given as follows:

$$
f^{*}(x \mid \underline{\boldsymbol{x}})=\sum_{q=0}^{\rho-1} C_{q} \frac{\alpha}{x} \exp \left[-\alpha \varpi_{q}\left(\log x-\log x_{j}\right)\right],
$$

where $C_{q}=\frac{(-1)^{q}\left(\begin{array}{c}\rho-1 \\ q\end{array}\right) R_{j}^{*} !}{(\boldsymbol{\rho}-\mathbf{1}) !\left(R_{j}^{*}-\boldsymbol{\rho}\right) ! \boldsymbol{q} !}$ and $\varpi_{q}=q+R_{j}^{*}-\rho+1$ for $q=0,1, \ldots, \rho-1$.
Upon combining (3.4) and (4.2), the Bayesian predictive density function of $X_{\rho: R_{j}^{*}}$, given progressive Type-II censoring scheme, is obtained as

$$
\begin{aligned}
f^{*}(x \mid \underline{\boldsymbol{x}}) & =I^{-1} \sum_{q=0}^{\rho-1} C_{q} \int_{0}^{x_{0}} \int_{0}^{\infty} \frac{\alpha^{D^{*}+\boldsymbol{a}+\mathbf{1}}}{\beta x} \\
& \times \exp \{-\alpha[W-(n+b) \log \beta+\log c]\} \\
& \times \exp \left[-\alpha \varpi_{q}\left(\log x-\log x_{j}\right)\right] d \alpha d \beta \\
& =\frac{I^{-1} \Gamma\left(D^{*}+a+1\right)}{(n+b)} \sum_{q=0}^{\rho-1} \frac{C_{q}}{x}\left[W(\underline{x})-(n+b) \log x_{0}\right. \\
& \left.+\log c+\varpi_{q}\left(\log x-\log x_{j}\right)\right]^{-\left(D^{*}+a+1\right)}
\end{aligned}
$$

The Bayesian predictive survival function of $X_{\rho: R_{j}^{*}}$, given Type-II PHCS, is given as

$$
\begin{aligned}
\bar{F}^{*}(t \mid \underline{x}) & =\int_{t}^{\infty} f^{*}(x \mid \underline{x}) d x \\
& =\frac{I^{-1} \Gamma\left(D^{*}+a\right)}{(n+b)} \sum_{q=0}^{\rho-1} \frac{C_{q}}{\varpi_{q}} \times\left[W(\underline{x})-(n+b) \log x_{0}\right. \\
& \left.+\log c+\varpi_{q}\left(\log t-\log x_{j}\right)\right]^{-\left(D^{*}+a\right)}
\end{aligned}
$$

The Bayesian point predictor of $X$ under the squared error loss function is the mean of the predictive density, given by

$$
\hat{X}_{\boldsymbol{\rho}: \boldsymbol{R}_{\boldsymbol{k}}}=\int_{0}^{\infty} x f^{*}(x \mid \underline{\boldsymbol{x}}) d x,
$$

where $f^{*}(x \mid \underline{\boldsymbol{x}})$ is given as in (4.3). The Bayesian predictive bounds of $100(1-\gamma) \%$ two-sided equi-tailed (ET) interval for $X_{\rho: R_{j}^{*}}$ can be obtained by solving the following two equations:

$$
\bar{F}^{*}\left(L_{E T} \mid \underline{\boldsymbol{x}}\right)=\frac{\gamma}{2} \text { and } \bar{F}^{*}\left(U_{E T} \mid \underline{\boldsymbol{x}}\right)=1-\frac{\gamma}{2}
$$

where $\bar{F}^{*}(t \mid \underline{x})$ is given as in (4.4), and $L_{E T}$ and $U_{E T}$ denote the lower and upper bounds, respectively. On the other hand, for the highest posterior density (HPD) method, the following two equations need to be solved:

$$
\bar{F}^{*}\left(L_{H P D} \mid \underline{\boldsymbol{x}}\right)-\bar{F}^{*}\left(U_{H P D} \mid \underline{\boldsymbol{x}}\right)=1-\gamma
$$

and

$$
f^{*}\left(L_{H P D} \mid \underline{x}\right)-f^{*}\left(U_{H P D} \mid \underline{x}\right)=0,
$$

where $f^{*}(x \mid \underline{x})$ is as in (4.3), and $L_{H P D}$ and $U_{H P D}$ denote the HPD lower and upper bounds, respectively.

\section{TWO-SAMPLE BAYESIAN PREDICTION}

Let $Y_{1: \ell: N}, Y_{2: \ell: N}, \ldots, Y_{\ell: \ell: N} \quad$ be a future independent progressively Type-II censored sample from the same population with censoring scheme $\underline{\boldsymbol{S}}=\left(S_{1}, S_{2}, \ldots, S_{\ell}\right)$. We develop here a general procedure for deriving the point and interval predictions for $Y_{s: \ell: N}$, based on the observed generalized PHCS. It is well known that the marginal density function of the $s^{t h}$ order statistic from a sample of size $N$ from a continuous distribution with $(C D F) F(x)$ and $(P D F) f(x)$ is given, see [12], by

$f_{Y_{S: \ell: N}}\left(y_{S} \mid \theta\right)=f\left(y_{S} \mid \theta\right)$ 


$$
=c(N, s) \sum_{q=0}^{s-1} C_{q, s}\left[\bar{F}\left(y_{s}\right)\right]^{T_{q, s^{-1}}} f\left(y_{s}\right)
$$

where $1 \leq s \leq \ell, c(N, s)=N\left(N-S_{1}-1\right) \ldots\left(N-S_{1}-\cdots-\right.$ $\left.S_{s-1}+1\right), \quad T_{q, s}=N-S_{1}-\cdots-S_{s-q-1}-s+q+1$ and $C_{q, s}=(-1)^{q}\left\{\frac{\left[\prod_{u=1}^{q} \sum_{v=s-q}^{s-q-u-1}\left(S_{v}+1\right)\right]}{\left[\prod_{u=1}^{s-q-1} \sum_{v=u}^{s-q-1}\left(S_{v}+1\right)\right]}\right\}$.

Upon substituting (1.1) and (1.2) in (5.1), the marginal density function of $Y_{s: l: N}$ is then obtained as

$f\left(y_{s} \mid \theta\right)$

$=c(N, s) \sum_{q=0}^{s-1} C_{q, s-1} \frac{\alpha}{y_{s}} \exp \left\{-\alpha\left[T_{q, s} \log \left(\frac{y_{s}}{\beta}\right)\right]\right\}$.

Upon combining (3.4) and (5.2), given Type-II PHCS , the Bayesian predictive density function of $Y_{S: \ell: N}$ is obtained as

$$
f^{*}\left(y_{s} \mid \underline{\boldsymbol{x}}\right)=\left\{\begin{array}{rr}
f_{1}^{*}\left(y_{s} \mid \underline{\boldsymbol{x}}\right) & 0<y_{s} \leq x_{0} \\
f_{2}^{*}\left(y_{s} \mid \underline{\boldsymbol{x}}\right) & y_{s}>x_{0}
\end{array}\right.
$$

where

$$
\begin{aligned}
& f_{1}^{*}\left(y_{s} \mid \underline{\boldsymbol{x}}\right)=\int_{0}^{y_{s}} \int_{0}^{\infty} f\left(y_{s} \mid \underline{\boldsymbol{x}}\right) \pi^{*}(\alpha, \beta ; \underline{\boldsymbol{x}}) d \alpha d \beta \\
&=I^{-1} c(N, s) \Gamma\left(D^{*}+a+1\right) \sum_{q=0}^{\rho-1} \frac{C_{q, s}}{\left(n+b+T_{q, s}\right)} \\
& \times\left[W(\underline{\boldsymbol{x}})-(n+b) \log y_{s}+\log c\right]^{-\left(D^{*}+a+1\right)} \\
& f_{2}^{*}\left(y_{s} \mid \underline{\boldsymbol{x}}\right)=\int_{0}^{x_{0}} \int_{0}^{\infty} f\left(y_{s} \mid \underline{\boldsymbol{x}}\right) \pi^{*}(\alpha, \beta ; \underline{\boldsymbol{x}}) d \alpha d \beta \\
&=I^{-1} c(N, s) \Gamma\left(D^{*}+a+1\right) \sum_{q=0}^{\rho-1} \frac{C_{q, s}}{\left(n+b+T_{q, s}\right)}[W(\underline{\boldsymbol{x}}) \\
&\left.-\left(n+b+T_{q, s}\right) \log x_{0}+T_{q, s} \log y_{s}+\log c\right]^{-\left(D^{*}+a+1\right)} .
\end{aligned}
$$

From (5.3), we simply obtain the predictive survival function of $Y_{s: \ell: N}$, given generalized PHCS Type-II, as

$$
\bar{F}^{*}(t \mid \underline{\boldsymbol{x}})=\int_{t}^{\infty} f^{*}\left(y_{s} \mid \underline{\boldsymbol{x}}\right) d y_{s}=\left\{\begin{array}{lr}
\bar{F}_{1}^{*}(t \mid \underline{\boldsymbol{x}}) & 0<t \leq x_{0}, \\
\bar{F}_{2}^{*}(t \mid \underline{\boldsymbol{x}}) & t>x_{0},
\end{array}\right.
$$

where

$$
\begin{gathered}
\bar{F}_{1}^{*}(t \mid \underline{\boldsymbol{x}})=\int_{0}^{x_{0}} f_{1}^{*}\left(y_{s} \mid \underline{\boldsymbol{x}}\right) d y_{s}+\int_{x_{0}}^{\infty} f_{2}^{*}\left(y_{s} \mid \underline{\boldsymbol{x}}\right) d y_{s} \\
=I^{-1} c(N, s) \Gamma\left(D^{*}+a\right) \sum_{q=0}^{\rho-1} \frac{C_{q, s}}{\left(n+b+T_{q, s}\right)(n+b) T_{q, s}} \\
\times\left\{\left(n+b+T_{q, s}\right)\left[W(\underline{\boldsymbol{x}})-(n+b) \log x_{0}+\log c\right]^{-\left(D^{*}+a\right)}\right. \\
T_{q, s}\left[W_{2}(\underline{\boldsymbol{x}})-(n+b) \log t+\log c\right]^{-\left(D^{*}+a\right)}
\end{gathered}
$$

and

$$
\begin{aligned}
\bar{F}_{2}^{*}(t \mid \underline{\boldsymbol{x}})=\int_{t}^{\infty} f_{2}^{*}\left(y_{s} \mid \underline{\boldsymbol{x}}\right) d y_{s} & \\
=I^{-1} c(N, s) \Gamma\left(D^{*}+a\right) \sum_{q=0}^{\rho-1} \frac{C_{q, s}}{\left(n+b+T_{q, s}\right) T_{q, s}} & {[W(\underline{\boldsymbol{x}})} \\
& +T_{q, s} \log t-\left(n+b+T_{q, s}\right) \log x_{0} \\
& +\log c]^{-\left(D^{*}+a\right)}
\end{aligned}
$$

The Bayesian point predictor of $Y_{s: \ell: N}, 1 \leq s \leq m$, under the squared error loss function is the mean of the predictive density, given by

$$
\hat{Y}_{S: \ell: N}=\int_{0}^{\infty} y_{S} f_{Y_{S:: N N}^{*}}^{*}\left(y_{S} \mid \underline{\boldsymbol{x}}\right) d y_{s}
$$

where $f_{Y_{s:: N}}^{*}\left(y_{S} \mid \underline{\boldsymbol{x}}\right)$ is given as in (5.3).

The Bayesian predictive bounds of $100(1-\gamma) \%$ ET interval for $Y_{s: t: N}, 1 \leq s \leq m$, can be obtained by solving the following two equations:

$$
\bar{F}_{S: \ell: N}^{*}\left(L_{E T} \mid \underline{\boldsymbol{x}}\right)=\frac{\gamma}{2} \text { and } \bar{F}_{S: \ell: N}^{*}\left(U_{E T} \mid \underline{\boldsymbol{x}}\right)=1-\frac{\gamma}{2},
$$

where $\bar{F}_{\text {s:::N }}^{*}(t \mid \underline{\boldsymbol{x}})$ is given as in (5.6), and $L_{E T}$ and $U_{E T}$ denote the lower and upper bounds, respectively. For the HPD method, the following two equations need to be solved:

$$
\bar{F}_{S: \ell: N}^{*}\left(L_{H P D} \mid \underline{\boldsymbol{x}}\right)-\bar{F}_{S: \ell: N}^{*}\left(U_{H P D} \mid \underline{\boldsymbol{x}}\right)=1-\gamma
$$

and

$$
f_{S: \ell: N}^{*}\left(L_{H P D} \mid \underline{\boldsymbol{x}}\right)-f_{S: \ell: N}^{*}\left(U_{H P D} \mid \underline{\boldsymbol{x}}\right)=0,
$$

where $f^{*}\left(y_{S} \mid \underline{\boldsymbol{x}}\right)$ is as in (5.3), and $L_{H P D}$ and $U_{H P D}$ denote the HPD lower and upper bounds, respectively.

\section{NUMERICAL RESULTS}

By using the algorithms which suggested in [13], we generated Type-II progressive hybrid censoring data from a sample of size $n=20$. The generated data from Pareto distribution (with $\alpha=3$ and $\beta=6$ ), using different types of Type-II PHCS are as follows:

1. Scheme 1: Suppose $m=8, T=7$ and $R=$ $(0,3,0,0,3,0,0,6)$. Since $T<X_{8: 8: 20}$, then the experiment would have terminated at $X_{8: 8: 20}=$ 8.088 with $R^{*}=R=(0,3,0,0,3,0,0,6), R_{\tau}^{*}=0$, and we would have the following data: $6.000,6.094$, $6.118,6.243,6.278,7.227,7.739$, and 8.088.

2. Scheme 2: Suppose $m=8$ and $T=9$ with the same censoring scheme in Scheme 1. Since $X_{8: 8: 20}<T$, then the experiment would have terminated at $T=9$ with $R^{*}=(0,3,0,0,3,0,0,0,5)$, $R_{\tau}^{*}=5, D=9$, and we have the following data: $6.000,6.094,6.118,6.243,6.278,7.227,7.739$, 8.088, and 8.398.

3. Scheme 3: Suppose $m=9, T=8$ and $R=$ $(2,0,0,2,0,0,2,0,5)$. Since $T<X_{9: 9: 20}$, then the experiment would have terminated at $X_{9: 9: 20}=$ 32.52 with $R^{*}=R=(2,0,0,2,0,0,2,0,5), R_{\tau}^{*}=0$, and we would have the following data: 6.096, 6.096 $6.210,6.234,6.382,6.426,7.293,7.887$, and 8.304.

4. Scheme 4: Suppose $m=9$ and $T=10$ with the same censoring scheme in Scheme 3. Since $X_{9: 9: 20}<T$, then the experiment would have terminated at $\quad T=10 \quad$ with $R^{*}=(2,0,0,2,0,0,2,0,0,0,3), R_{\tau}^{*}=5, D=11$, and we have the following data: 6.096, 6.096, 6.210, $6.234,6.382,6.426,7.293,7.887,8.304,8.621$, and 9.825 .

We assume these data to have come from Pareto distribution with $\alpha$ and $\beta$ are unknown. Based on the above generated Type-II HPCS, Table 1 presents the ML and Bayesian estimates of $\alpha$ and $\beta$. Table 2 presents the point predictor and 
$95 \%$ Bayesian prediction bounds of $X_{\rho: R_{j}^{*}}$ based on the above generated Type-II HPCS and Table 3 presents the point predictor and 95\% Bayesian prediction bounds of $Y_{S: \ell: N}$ from the future progressive censored sample of size $\ell=10$ from a sample of size $N=20$ with progressive censoring scheme $S=(1,1,0,0,2,1,0,2,1,2)$ based on the above generated TypeII PHCS. All these results in Tables 1-3 are computed based on two different choices of the hyperparameters $(a, b, c, d)$, namely,

1. $a=1, b=0.55, c=5.2 \quad$ and $\quad d=11.03$ : informative prior $(I P)$.

2. $a=-1, b=0, c=1$ and $=\infty$ : noninformative prior $(N I P)$.
Table 1: The ML and Bayesian estimates of $\alpha$ and $\beta$.

\begin{tabular}{|c|c|c|c|c|c|c|}
\hline \multirow[b]{2}{*}{ Scheme } & \multirow[b]{2}{*}{$\hat{\alpha}_{M L}$} & \multicolumn{2}{|c|}{$\hat{\alpha}_{B}$} & \multirow[b]{2}{*}{$\hat{\beta}_{M L}$} & \multicolumn{2}{|c|}{$\hat{\beta}_{B}$} \\
\hline & & $I P$ & NIP & & $I P$ & NIP \\
\hline 1 & 2.823 & 2.574 & 2.470 & 6.000 & 5.875 & 5.862 \\
\hline 2 & 2.987 & 2.748 & 2.608 & 6.137 & 5.903 & 5.932 \\
\hline 3 & 3.175 & 2.866 & 2.823 & 6.096 & 5.983 & 5.975 \\
\hline 4 & 3.014 & 2.985 & 3.154 & 6.074 & 6.098 & 6.154 \\
\hline
\end{tabular}

Table 2: Bayesian point predictor and 95\% ET and HPD prediction intervals for $X_{\rho: R_{j}^{*}}$ for $\rho=1, \ldots, R_{j}^{*}, k=1, \ldots, D^{*}$ or $\tau$.

\begin{tabular}{|c|c|c|c|c|c|c|c|c|}
\hline \multirow{2}{*}{ Scheme } & \multirow{2}{*}{$R_{j}^{*}$} & \multirow[t]{2}{*}{$\rho$} & \multicolumn{3}{|c|}{$G P$} & \multicolumn{3}{|c|}{$J P$} \\
\hline & & & $X_{\rho: R_{j}^{*}}$ & ET interval & HPD interval & $X_{\rho: R_{j}^{*}}$ & ET interval & HPD interval \\
\hline \multirow[t]{12}{*}{1} & 2 & 1 & 7.170 & $(6.114,10.999)$ & $(6.094,9.657)$ & 7.339 & $(6.115,11.735)$ & $(6.094,10.092)$ \\
\hline & & 2 & 9.520 & $(6.324,19.891)$ & $(30.906,83.317)$ & 11.455 & $(6.331,22.996)$ & $(6.101,17.567)$ \\
\hline & & 3 & 36.538 & $(6.909,68.931)$ & $(6.021,43.964)$ & 40.197 & $(6.930,93.766)$ & $(6.032,54.743)$ \\
\hline & 5 & 1 & 7.386 & $(6.299,11.331)$ & $(6.278,9.948)$ & 7.560 & $(6.300,12.090)$ & $(6.278,10.397)$ \\
\hline & & 2 & 9.807 & $(6.515,20.492)$ & $(6.287,16.342)$ & 11.783 & $(6.522,23.690)$ & $(6.285,18.097)$ \\
\hline & & 3 & 37.442 & $(7.118,71.012)$ & $(6.203,45.292)$ & 41.199 & $(7.140,96.598)$ & $(6.214,56.396)$ \\
\hline & 8 & 1 & 8.732 & $(8.101,10.866)$ & $(8.088,10.181)$ & 8.794 & $(8.102,11.224)$ & $(8.088,10.408)$ \\
\hline & & 2 & 9.594 & $(8.223,13.541)$ & $(8.097,12.288)$ & 9.765 & $(8.227,14.431)$ & $(8.096,12.851)$ \\
\hline & & 3 & 10.840 & $(8.465,17.601)$ & $(8.014,15.334)$ & 11.270 & $(8.474,19.500)$ & $(8.021,16.504)$ \\
\hline & & 4 & 12.912 & $(8.843,24.984)$ & $(8.329,20.737)$ & 14.354 & $(8.857,29.158)$ & $(8.305,23.165)$ \\
\hline & & 5 & 17.891 & $(9.440,43.067)$ & $(8.539,32.769)$ & 26.746 & $(9.461,54.375)$ & $(8.491,38.766)$ \\
\hline & & 6 & 56.669 & $(10.575,143.208)$ & $(8.756,87.896)$ & 64.721 & $(10.611,212.151)$ & $(8.674,116.779)$ \\
\hline \multirow[t]{11}{*}{2} & 2 & 1 & 7.208 & $(6.115,11.160)$ & $(6.094,9.786)$ & 7.344 & $(6.116,11.849)$ & $(6.094,10.205)$ \\
\hline & & 2 & 9.592 & $(6.336,20.377)$ & $(6.103,16.253)$ & 10.734 & $(6.345,23.249)$ & $(6.102,17.874)$ \\
\hline & & 3 & 23.316 & $(6.956,72.188)$ & $(6.017,46.103)$ & 26.498 & $(6.986,95.345)$ & $(6.026,56.487)$ \\
\hline & 5 & 1 & 7.426 & $(6.300,11.497)$ & $(6.278,10.082)$ & 7.565 & $(6.301,12.207)$ & $(6.278,10.513)$ \\
\hline & & 2 & 9.881 & $(6.527,20.992)$ & $(6.287,16.743)$ & 11.051 & $(6.536,23.951)$ & $(6.286,18.414)$ \\
\hline & & 3 & 34.182 & $(7.166,74.368)$ & $(6.198,47.495)$ & 42.168 & $(7.197,98.224)$ & $(6.208,58.192)$ \\
\hline & 9 & 1 & 9.245 & $(8.415,12.073)$ & $(8.398,11.159)$ & 9.324 & $(8.416,12.516)$ & $(8.398,11.443)$ \\
\hline & & 2 & 10.466 & $(8.579,16.023)$ & $(8.409,14.219)$ & 10.697 & $(8.585,17.202)$ & $(8.408,14.967)$ \\
\hline & & 3 & 12.468 & $(8.922,23.048)$ & $(8.511,19.415)$ & 13.148 & $(8.939,25.916)$ & $(8.501,21.150)$ \\
\hline & & 4 & 16.948 & $(9.514,40.300)$ & $(8.705,31.141)$ & 20.757 & $(9.546,48.609)$ & $(8.678,35.744)$ \\
\hline & & 5 & 49.791 & $(10.679,138.006)$ & $(8.032,85.785)$ & 55.274 & $(10.742,192.265)$ & $(8.061,109.630)$ \\
\hline \multirow[t]{11}{*}{3} & 1 & 1 & 7.634 & $(6.123,13.275)$ & $(6.096,11.211)$ & 7.817 & $(6.123,13.983)$ & $(6.096,11.604)$ \\
\hline & & 2 & 16.555 & $(6.460,40.972)$ & $(6.103,28.401)$ & 27.276 & $(6.462,47.278)$ & $(6.102,31.330)$ \\
\hline & 4 & 1 & 7.807 & $(6.262,13.575)$ & $(6.234,11.465)$ & 7.994 & $(6.262,14.300)$ & $(6.234,11.866)$ \\
\hline & & 2 & 16.909 & $(6.606,41.900)$ & $(6.241,29.044)$ & 27.776 & $(6.609,48.348)$ & $(6.240,32.039)$ \\
\hline & 7 & 1 & 9.133 & $(7.325,15.881)$ & $(7.293,13.413)$ & 9.347 & $(7.326,16.729)$ & $(7.293,13.882)$ \\
\hline & & 2 & 19.612 & $(7.728,49.018)$ & $(7.302,33.978)$ & 31.566 & $(7.731,56.561)$ & $(7.300,37.482)$ \\
\hline & 9 & 1 & 9.010 & $(8.319,11.336)$ & $(8.304,10.596)$ & 9.347 & $(7.326,16.729)$ & $(7.293,13.882)$ \\
\hline & & 2 & 10.006 & $(8.457,14.451)$ & $(8.314,13.045)$ & 10.114 & $(8.458,15.083)$ & $(8.313,13.433)$ \\
\hline & & 3 & 11.579 & $(8.747,19.736)$ & $(8.219,16.980)$ & 11.869 & $(8.747,21.214)$ & $(8.226,17.863)$ \\
\hline & & 4 & 14.775 & $(7.769,31.869)$ & $(8.583,25.565)$ & 16.121 & $(9.239,35.807)$ & $(8.556,27.743)$ \\
\hline & & 5 & 36.075 & $(10.204,91.576)$ & $(8.797,61.068)$ & 47.259 & $(10.192,112.466)$ & $(8.747,70.597)$ \\
\hline 4 & 1 & 1 & 7.608 & $(6.123,13.102)$ & $(6.096,11.139)$ & 7.700 & $(6.124,13.600)$ & $(6.096,11.426)$ \\
\hline
\end{tabular}




$\begin{array}{cccccccc} & 2 & 14.399 & (6.468,39.296) & (6.104,27.748) & 17.782 & (6.471,43.479) & (6.103,29.797) \\ 4 & 1 & 7.780 & (6.262,13.399) & (6.234,11.392) & 7.874 & (6.262,13.908) & (6.234,11.685) \\ & 2 & 14.719 & (6.614,40.186) & (6.242,28.376) & 18.157 & (6.618,44.463) & (6.241,30.471) \\ 7 & 1 & 9.102 & (7.326,15.675) & (7.293,13.327) & 9.211 & (7.326,16.271) & (7.293,13.670) \\ & 2 & 17.170 & (7.738,47.012) & (7.303,33.197) & 21.026 & (7.742,52.017) & (7.301,35.647) \\ 11 & 1 & 11.305 & (9.854,16.363) & (9.825,14.685) & 11.373 & (9.855,16.775) & (9.825,14.936) \\ & 2 & 14.175 & (10.165,27.014) & (9.841,22.487) & 14.479 & (10.169,28.568) & (9.840,23.392) \\ & 3 & 27.433 & (11.038,77.634) & (9.691,54.000) & 34.490 & (11.045,87.422) & (9.702,58.795)\end{array}$

Table 3: Bayesian point predictor and 95\% ET and HPD prediction intervals $Y_{s: \ell: N}$ for $s=1, \ldots, \ell$.

\begin{tabular}{|c|c|c|c|c|c|c|c|}
\hline \multirow{2}{*}{ Scheme } & \multirow[t]{2}{*}{$s$} & \multicolumn{3}{|c|}{$G P$} & \multicolumn{3}{|c|}{$J P$} \\
\hline & & $\widehat{Y}_{S: \ell: N}$ & ET interval & HPD interval & $\widehat{Y}_{S: \ell: N}$ & ET interval & HPD interval \\
\hline \multirow[t]{10}{*}{1} & 1 & 6.007 & $(5.612,6.431)$ & $(5.601,6.419)$ & 6.004 & $(5.563,6.472)$ & $(5.551,6.459)$ \\
\hline & 2 & 6.149 & $(5.717,6.776)$ & $(5.673,6.715)$ & 6.158 & $(5.682,6.865)$ & $(5.628,6.791)$ \\
\hline & 3 & 6.303 & $(5.819,7.136)$ & $(5.748,7.020)$ & 6.326 & $(5.796,7.286)$ & $(5.709,7.137)$ \\
\hline & 4 & 6.525 & $(5.937,7.704)$ & $(5.833,7.485)$ & 6.569 & $(5.925,7.954)$ & $(5.799,7.669)$ \\
\hline & 5 & 6.776 & $(6.051,8.344)$ & $(5.921,8.006)$ & 6.847 & $(6.045,8.722)$ & $(5.891,8.274)$ \\
\hline & 6 & 7.064 & $(5.822,9.090)$ & $(6.011,8.607)$ & 7.167 & $(6.162,9.633)$ & $(5.983,8.981)$ \\
\hline & 7 & 7.488 & $(6.303,10.289)$ & $(6.107,9.549)$ & 7.645 & $(6.303,11.114)$ & $(6.081,10.100)$ \\
\hline & 8 & 8.012 & $(6.462,11.828)$ & $(6.203,10.741)$ & 8.251 & $(6.464,13.061)$ & $(6.177,11.543)$ \\
\hline & 9 & 8.684 & $(6.651,13.908)$ & $(6.299,12.320)$ & 9.056 & $(6.653,15.758)$ & $(6.270,13.491)$ \\
\hline & 10 & 23.635 & $(7.241,59.313)$ & $(6.357,38.681)$ & 44.040 & $(7.252,79.238)$ & $(6.320,47.588)$ \\
\hline \multirow[t]{10}{*}{2} & 1 & 6.007 & $(5.601,6.444)$ & $(5.589,6.431)$ & 6.004 & $(5.553,6.482)$ & $(5.541,6.469)$ \\
\hline & 2 & 6.154 & $(5.708,6.795)$ & $(5.663,6.735)$ & 6.163 & $(5.673,6.878)$ & $(5.621,6.806)$ \\
\hline & 3 & 6.314 & $(5.812,7.161)$ & $(5.742,7.047)$ & 6.336 & $(5.789,7.298)$ & $(5.704,7.156)$ \\
\hline & 4 & 6.544 & $(5.935,7.738)$ & $(5.830,7.524)$ & 6.586 & $(5.921,7.964)$ & $(5.797,7.694)$ \\
\hline & 5 & 6.804 & $(6.054,8.387)$ & $(5.922,8.056)$ & 6.872 & $(6.048,8.726)$ & $(5.894,8.304)$ \\
\hline & 6 & 7.103 & $(6.173,9.143)$ & $(6.016,8.671)$ & 7.201 & $(6.172,9.628)$ & $(5.991,9.015)$ \\
\hline & 7 & 7.542 & $(6.320,10.361)$ & $(6.118,9.637)$ & 7.691 & $(6.323,11.097)$ & $(6.096,10.144)$ \\
\hline & 8 & 8.087 & $(6.490,11.925)$ & $(6.222,10.859)$ & 8.307 & $(6.497,13.022)$ & $(6.200,11.595)$ \\
\hline & 9 & 8.785 & $(6.693,14.041)$ & $(6.326,12.482)$ & 9.116 & $(6.702,15.684)$ & $(6.303,13.556)$ \\
\hline & 10 & 23.220 & $(7.322,61.976)$ & $(6.385,40.435)$ & 38.452 & $(7.348,5.520)$ & $(6.355,48.960)$ \\
\hline \multirow[t]{10}{*}{3} & 1 & 6.102 & $(5.747,6.481)$ & $(5.738,6.471)$ & 6.099 & $(5.716,6.501)$ & $(5.707,6.492)$ \\
\hline & 2 & 6.229 & $(5.841,6.783)$ & $(5.803,6.733)$ & 6.232 & $(5.818,6.830)$ & $(5.776,6.773)$ \\
\hline & 3 & 6.367 & $(5.932,7.095)$ & $(5.872,7.000)$ & 6.377 & $(5.917,7.175)$ & $(5.848,7.062)$ \\
\hline & 4 & 6.565 & $(6.039,7.582)$ & $(5.950,7.404)$ & 6.585 & $(6.029,7.716)$ & $(5.928,7.502)$ \\
\hline & 5 & 6.787 & $(6.143,8.124)$ & $(6.032,7.852)$ & 6.819 & $(5.070,8.326)$ & $(6.011,7.994)$ \\
\hline & 6 & 7.040 & $(6.246,8.748)$ & $(6.115,8.363)$ & 7.087 & $(6.241,9.036)$ & $(6.095,8.561)$ \\
\hline & 7 & 7.408 & $(6.374,9.738)$ & $(6.204,9.156)$ & 7.480 & $(6.368,10.170)$ & $(6.184,9.444)$ \\
\hline & 8 & 7.859 & $(6.521,10.986)$ & $(6.295,10.144)$ & 7.965 & $(6.513,11.618)$ & $(6.273,10.556)$ \\
\hline & 9 & 8.428 & $(6.695,12.638)$ & $(6.386,11.431)$ & 8.586 & $(6.684,13.564)$ & $(6.360,12.022)$ \\
\hline & 10 & 16.889 & $(7.231,45.147)$ & $(6.448,31.318)$ & 22.536 & $(7.216,52.912)$ & $(6.417,35.003)$ \\
\hline \multirow[t]{7}{*}{4} & 1 & 6.102 & $(5.750,6.477)$ & $(5.741,6.467)$ & 6.099 & $(5.725,6.491)$ & $(5.717,6.483)$ \\
\hline & 2 & 6.229 & $(5.841,6.769)$ & $(5.807,6.724)$ & 6.230 & $(5.822,6.802)$ & $(5.784,6.753)$ \\
\hline & 3 & 6.366 & $(5.932,7.070)$ & $(5.876,6.984)$ & 6.373 & $(5.917,7.125)$ & $(5.856,7.029)$ \\
\hline & 4 & 6.563 & $(6.038,7.537)$ & $(5.955,7.378)$ & 6.577 & $(6.029,7.629)$ & $(5.937,7.448)$ \\
\hline & 5 & 6.784 & $(6.144,8.054)$ & $(6.038,7.812)$ & 6.807 & $(6.137,8.192)$ & $(6.021,7.912)$ \\
\hline & 6 & 7.034 & $(6.250,8.646)$ & $(6.124,8.304)$ & 7.069 & $(6.245,8.8415)$ & $(6.108,8.444)$ \\
\hline & 7 & 7.399 & $(6.382,9.585)$ & $(6.217,9.068)$ & 7.451 & $(6.3781,9.8752)$ & $(6.202,9.270)$ \\
\hline
\end{tabular}




$\begin{array}{ccccccc}8 & 7.844 & (6.535,10.760) & (6.314,10.016) & 7.920 & (6.5304,11.1822) & (6.297,10.302) \\ 9 & 8.405 & (6.716,12.306) & (6.411,11.244) & 8.514 & (6.7104,12.9191) & (6.393,11.651) \\ 10 & 15.539 & (7.273,42.984) & (6.477,30.416) & 17.871 & (7.2660,48.0193) & (6.455,32.935)\end{array}$

\section{CONCLUSIONS AND DISCUSSION}

In this paper, based on Type-II hybrid progressive censored scheme the ML and Bayesian estimators of the unknown parameters for Pareto distribution has been developed. Also, the problem for deriving one- and two-sample Bayesian prediction have been discussed.

From the results in Tables 2 and 3, we notice that the point predictor of the unknown parameters is between the lower and upper bounds of the prediction intervals. Moreover, a comparison of the results for the informative priors with the corresponding ones for non-informative priors reveals that the former produce more precise results, as we would expect. Finally, the HPD prediction intervals seem to be more precise than the ET prediction intervals.

\section{REFERENCES}

[1] Aggarwala, R., \& Balakrishnan, N. (1998). Some properties of progressive censored order statistics from arbitrary and uniform distributions with applications to inference and simulation. Journal of statistical planning and inference, 70(1), 35-49.

[2] Raqab, M. Z., Asgharzadeh, A., \& Valiollahi, R. (2010) Prediction for Pareto distribution based on progressively Type-II censored samples. Computational Statistics \& Data Analysis, 54(7), 1732-1743.

[3] Balakrishnan, N., \& Cohen, A. C. (2014). Order statistics $\&$ inference: estimation methods. Elsevier

[4] Childs, A., Chandrasekar, B., Balakrishnan, N., \& Kundu, D. (2003). Exact likelihood inference based on Type-I and Type-II hybrid censored samples from the exponential distribution. Annals of the Institute of Statistical Mathematics, 55(2), 319-330.

[5] Pareto, V., (1897). Cours d'Economie Politique. Rouge et Cie, Paris.

[6] Davis, H. T., \& Feldstein, M. L. (1979). The generalized Pareto law as a model for progressively censored survival data. Biometrika, 66(2), 299-306.

[7] Cohen, A. C., \& Whitten, B. J. (1988). Parameter estimation in reliability and life span models. M. Dekker.

[8] Grimshaw, S. D. (1993). Computing maximum likelihood estimates for the generalized Pareto distribution. Technometrics, 35(2), 185-191

[9] Lwin, T. (1972). Estimation of the tail of the Paretian law Scandinavian Actuarial Journal, 1972(2), 170-178

[10] Arnold, B. C., \& Press, S. J. (1989). Bayesian estimation and prediction for Pareto data. Journal of the American Statistical Association, 84(408), 1079-1084.

[11] Basak, I., Basak, P., \& Balakrishnan, N. (2006). On some predictors of times to failure of censored items in progressively censored samples. Computational statistics $\&$ data analysis, 50(5), 1313-1337.

[12] Balakrishnan, N., Childs, A., \& Chandrasekar, B. (2002). An efficient computational method for moments of order statistics under progressive censoring. Statistics \& probability letters, 60(4), 359-365.

[13] Balakrishnan, N., \& Aggarwala, R. (2000). Progressive censoring: theory, methods, and applications. Springer Science \& Business Media. 